\title{
Urine Analysis has a Very Broad Prospect in the Future
}

\author{
Zijuan Zhang ${ }^{1,2 \dagger}$, Jingnan Liu ${ }^{1,2 \dagger}$, Yaxing Cheng ${ }^{1,2}$, Jian Chen ${ }^{1}$, Huihui Zhao ${ }^{1,2 *}$ and \\ Xiaoqiao Ren ${ }^{1,2 *}$
}

${ }^{1}$ School of Traditional Chinese Medicine, Beijing University of Chinese Medicine, Beijing, China, ${ }^{2}$ Institute of National Medicine, Beijing University of Chinese Medicine, Beijing, China

Medical tests are playing an increasingly important role in the diagnosis and treatment of diseases. Urine tests, blood tests and stool tests together constitute the three major routine examination items of modern medicine and are an important part of medical tests. Urine is a body fluid normally metabolized by the human body. Compared with using blood as a test sample, using urine as a medical test sample has many advantages, such as noninvasiveness and convenient collection. This article discusses the advantages of urine test

OPEN ACCESS

Edited by:

Liang Qiao,

Fudan University, China

Reviewed by:

Kewei Ren,

University of Massachusetts Amherst,

United States

Donato Calabria,

Università di Bologna, Italy

*Correspondence:

Huihui Zhao

hh686@126.com

Xiaoqiao Ren

rxq23111111@126.com

tThese authors have contributed equally to this work and share first authorship

Specialty section:

This article was submitted to Biomedical Analysis and Diagnostics,

a section of the journal

Frontiers in Analytical Science

Received: 17 November 2021 Accepted: 29 December 2021

Published: 02 February 2022

Citation:

Zhang Z, Liu J, Cheng Y, Chen J, Zhao $H$ and Ren $X$ (2022) Urine Analysis has a Very Broad Prospect in the Future.

Front. Anal. Sci. 1:812301. doi: 10.3389/frans.2021.812301 compared with blood test, the understanding and application of urine in traditional medicine, the application of urine test in social life, the current dilemma and the future urine test may play a greater role The value and advantages are discussed, aiming to increase people's attention to urine testing by explaining the advantages of urine testing, and to discover more functions of urine testing, thereby optimizing medical testing methods and reducing the pain and fear of patients. Improve inspection efficiency, reduce national and personal medical inspection expenditures, and save medical resources.

Keywords: urine test, blood test, medical test, traditional Chinese medicine urinary examination, Tibetan medicine urinary examination

\section{INTRODUCTION}

Urine is the liquid excretion excreted through the urinary system and urinary tract for the needs of humans and vertebrates for metabolism. It contains key information about human health, dietary intake and exposure to environmental pollutants (Zha and Huang, 2019). Urine is the normal metabolic water of the human body, and the normal acquisition of urine will not have an adverse effect on the body, which is convenient for multiple sampling and dynamic observation and analysis of the patient's treatment status. Both urine and blood contain rich physiological and pathological information of the human body, but due to the particularity of blood itself, the use of blood as a medical test sample will cause more or less harm to the human body. With the continuous development of testing technology, some diseases in which urine is used to find markers are slowly being discovered. This will allow more diseases in the future to be easily and quickly tested through urine, and even at home, to be able to make diagnosis and assessment of the prognosis of treatment, and to make up for the inconvenience or delay of blood testing and pathological section examination. If the urine test can be used to diagnose the disease more accurately, it will save more medical costs, reduce the mental and economic burden of patients, and have important clinical practical significance. It is currently known that every compound found in urine can also be found in blood. More than 484 compounds have been identified in urine (either through experiments or through literature reviews), and have not been previously found in blood reported. Urine contains all 
the compounds in the blood, and at the same time has what the blood does not have. This may be related to the extraordinary work done by the kidneys in concentrating certain metabolites in the blood. For certain compounds, such as histamine, androsterone, norepinephrine, metatyramine, and aldosterone, the concentration difference between the two biological fluids is sometimes even more than 1,000 times. Therefore, although the amounts of water-soluble compounds in blood and urine are almost the same, the concentrations of these compounds are often very different. This difference, combined with the ability of the kidneys to process abnormally high or low concentrations of metabolites, makes urine a particularly useful biological fluid for medical diagnosis. According to data in UMDB (Urine Metabolome Database), urine metabolites have been used to describe nearly 220 diseases. In addition, the kidney's ability to filter toxins or foreign biological substances makes urine a particularly useful biological fluid for diet and drug monitoring, and to assess exposure to chemicals or pollutants (Bouatra et al., 2013). As shown in the figure below, Figures 1-3 introduce a concise summary of the urinalysis.

\section{COMPARED WITH BLOOD TESTS, URINE TESTS HAVE THE FOLLOWING ADVANTAGES}

\section{The Collection is Simple and Non-Invasive}

The urine can be collected completely non-invasively and continuously during the collection process, and the operation is simple, which is convenient for timely observation and analysis of the dynamics and prognosis of the disease. The blood collection is invasive, and the time for collecting blood samples, the operation process and even the position of blood collection have relatively strict requirements (Zhang and Liang, 2016). Blood is one of the most important tissues in the human body (Fan, 2020). A large amount of blood sampling will have adverse effects on the patient's body, and multiple blood sampling in a short period of time will affect the patient's physical and mental health, especially for children, the elderly, and patients with physical weakness. It is not conducive to timely assessment of the disease. Urine dynamic analysis is more convenient and safer than blood.

\section{Less Affected by the Regulation of Internal Mechanisms}

The human body has a tendency to maintain stability, and blood, as one of the important components of the human body, also has this characteristic. A biomarker is essentially a variable. Variables in the blood, liver, kidney, lung, skin and other organs will use various mechanisms to reduce this variable in the blood (Gao, 2013). Most of the changed information does not stay in the blood for a long time, but the changed information it is also the essence of disease markers. Urine does not have the necessary and mechanism to maintain stability. More types of disease information can be accumulated in urine, and many changes in the body can be reflected in urine, which is more conducive to finding markers in urine (Zha and Huang, 2019).

\section{Facilitate Continuous Monitoring}

Because of the research on Drosophila circadian clock genes and molecular regulation mechanisms, the three scientists won the Nobel Prize. Biological rhythms and time biological rhythms have once again attracted widespread attention. As early as 1959, the famous American physiologist Franz Halberg proposed the use of "circa-dian clock" to define a biological clock with a $24 \mathrm{~h}$ rhythm, and the term "chronobiology" to define interbiology. He tested the level of hormones in his urine by a friend of his for the past 30 years. It is found that the human body has a particularly obvious circadian rhythm, that is, a 24-h cycle of change, and it also has a clear 7-days rhythm. Rhythm changes in urine will also provide the possibility and convenience of disease dynamic monitoring. Blood and urine are rich in disease biological information. The requirements for obtaining urine and testing conditions are lower than those of blood, so if you use urine the continuous detection of liquid can control the disease, and it will be more convenient to observe and analyze the dynamics and prognosis of the disease in time (Yuan et al., 2018).

\section{Suitable for People who are Inconvenient to Draw Blood for Testing}

For children, people with special beliefs who are inconvenient to collect blood, and people who need long-term monitoring of diseases, the way of urine sampling and testing is much more friendly. Urine Legionella (Lp) antigen test card is used in the clinic to detect Legionella pneumophila in the urine of children, which can quickly diagnose the child as Legionella pneumophila infection, shorten the diagnosis time, improve the treatment efficiency, and reduce the number of children Resistance in routine testing (Qiu et al., 2020).

\section{Urine can be Collected in Large Quantities}

Urine can be collected continuously in large quantities and will not cause harm to the human body. Under suitable storage conditions, the stability of a variety of compounds in the urine is better. A large amount of urine can be collected to solve the problem that the content of certain components is too low and it is inconvenient to detect (Wang and Bao, 2019; Zha and Huang, 2019).

\section{The Samples are Easy to Store, Handle and Transport}

Compared with blood samples, urine samples have relatively low requirements for storage, transportation and processing conditions. Relatively reduce the consumption of manpower and material resources. The protein group in the urine can be stored for a long time without significant changes. Someone has proposed whether it is possible to save the urine information of the patient as a case by preserving the protein group in the urine (Zha and Huang, 2019). This may be it provides a new idea for the innovation of future case preservation. At the same time, the content of other proteins in the urine is low, and the detection background is low, which is conducive to the separation of DNA. 


\section{It can be Monitored Macroscopically}

Because urine can be visually observed, it is convenient to make timely judgments of diseases that can be detected from the macroscopic changes in urine, such as: the appearance or change of urine foam, turbidity, and sediment. Have a preliminary understanding of one's own situation, draw attention to it, and conduct timely examination, diagnosis and treatment of physical conditions to prevent the appearance or further aggravation of the disease. With the improvement of the level of health awareness, people's attention to urine is gradually increasing. In the theory of Chinese medicine, the color of urine is closely related to the cold and heat conditions in the body. People can understand their physical conditions through awareness of their own physical conditions and changes in urine. If you have not reached the disease state, through subjective feelings and objective urine-assisted observations, you can adjust your diet or living habits in a timely manner, and you can perform selfregulation simply and easily, which is convenient for keeping the body in a good state.

\section{Reduce the Risk of Medical Staff as Occupational Exposure}

For the blood collection of some patients with infectious diseases, urine testing can relatively reduce the risk of medical workers. Traditional blood test methods require invasive collection of samples, and there is a risk of needle stick injuries. Investigations and studies have found that the main types of exposure of medical staff are sharp object injuries, which mainly occur when sorting out medical equipment, medical waste and blood sampling (Zhang et al., 2020). It has been confirmed that more than 20 pathogens can enter the body through damaged skin and mucous membranes and cause infections of medical staff, such as Treponema pallidum, hepatitis B and C viruses, human immunodeficiency virus, etc., (Tang et al., 2020). Clinically, a large number of blood samples will increase the risk of infection among medical staff.

\section{Saving Medical Staff Time and Improving Medical Efficiency}

With the continuous development of urine testing automation technology and the emergence of automated urine instruments, the time for urine testing has been greatly reduced (Carrera and Bueno, 2020), because most patients can complete sample collection independently or with the assistance of family members. The workload of medical workers is reduced, the time cost of medical workers is saved, and the efficiency of medical inspection is improved.

\section{Test for HIV Through Urine}

In addition to HIV antibodies in the blood of HIV-infected persons, HIV antibodies can be detected in their urine, saliva, tears and other body fluids or secretions. The concentration of HIV-1 antibodies (mainly IgG) in urine and serum The antibody concentration in parallel (Dai et al., 2003). In a HIV blood and urine test of 203 people, it was found that the sensitivity and specificity of urine specimens to detect HIV-1 antibodies were similar to those of blood specimens (the sensitivity of blood specimens was $99.9 \%$, and the specificity was $99.9 \%$; urine specimens had a sensitivity of $99.9 \%$ and specificity of $99.9 \%$. The sensitivity of the specimen is $98.7 \%$, and the specificity is 99.1\%). Many studies have also reflected that the urine test for HIV also has good specificity and sensitivity (Ye et al., 2019; Ren, 2020). Studies have shown that in the HIV test of patients, the acceptance rate of urine test is higher than that of blood test and oral mucosal collection test. Urine test reduces patients' fear of medical tests (Gao, 2013). Compared with blood, it is easy to operate and suitable for large-scale screening and monitoring.

\section{Food Safety Testing in Urine}

With the development of social productivity, people's material life has become richer, but the food safety problem has become more and more serious. The people's pursuit of a healthy diet and the insecurity of the food consumer market constitute a pair of important contradictions in the market today. Hormones and tranquilizers are used in poultry to sedative and hypnotize poultry, gain weight, and use antibiotics in violation of regulations, so as to achieve illegal benefits. Large doses of drugs remaining in animal products enter the human body after being eaten. Serious harm to the human body, precocious puberty in children, obesity, and many chronic physical and mental diseases are becoming more and more serious, which are closely related to these food problems. In this regard, it is very important to strengthen the supervision of food safety. There have been many studies on children's urine testing and found that a variety of commonly used antibiotics and those limited to livestock and poultry can be detected in children's urine. For example: There was a survey of more than 1,000 children and found that 580 human urine has antibiotics, and some children even contain 6 kinds of antibiotics. Enrofloxacin and tylosin, which are generally restricted to livestock and poultry, were also detected in children (Hexing et al., 2015; Li et al., 2017; Wang et al., 2018). Strengthening supervision at the source link can not only reduce the chance of illness, but also reduce the use of medical resources and save social resources. At present, experiments have proved that the use of glucocorticoid drugs, diazepam and meat essence, antibiotics and their metabolites can be detected in the urine of pigs, and the urine is used to monitor poultry meat problems before slaughter, to avoid damage to the animal body and secondary infections and other problems.

\section{Urine Biomarkers Have the Advantage of Early Diagnosis of Diseases}

Urine biomarkers have the advantage of early diagnosis of diseases, because in the case of no obvious pathological symptoms in the early stage of the disease, the homeostasis mechanism effectively maintains the stability of the body's internal environment and removes harmful ones from the body through various methods. Keep the composition and characteristics of body fluids (especially blood) unchanged or within the normal range. Therefore, the early characteristics of many diseases are difficult to reflect in the blood. Compared with 


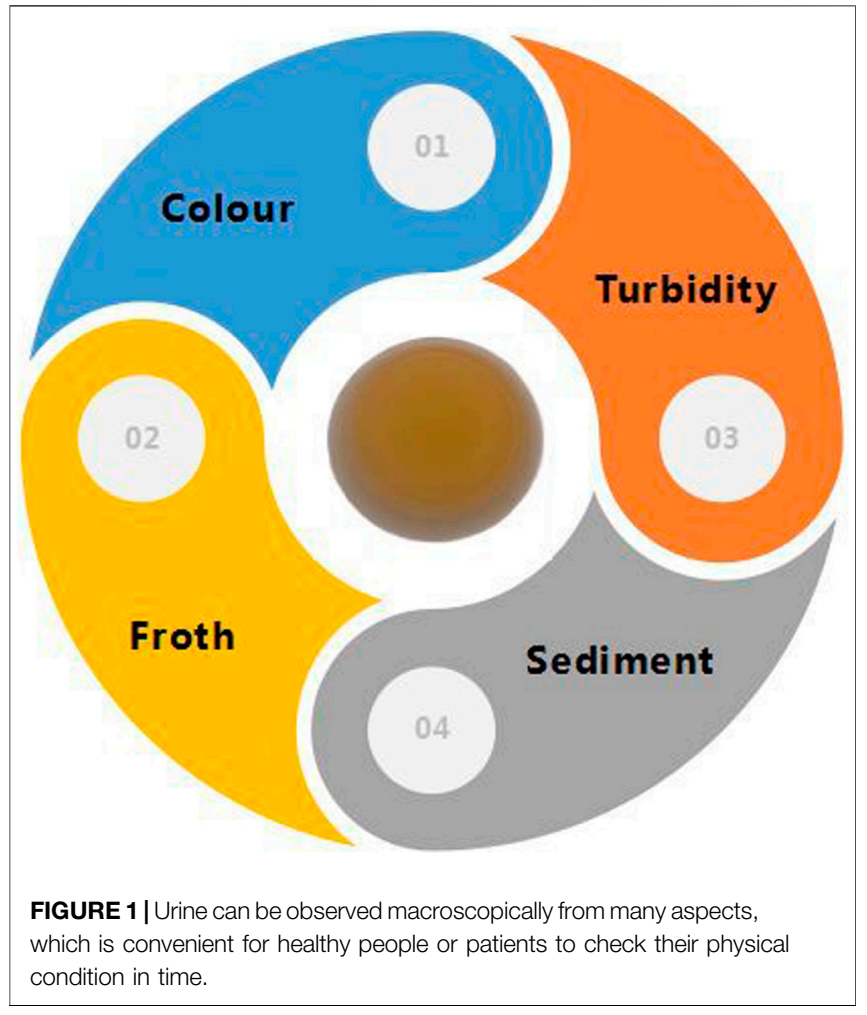

blood, urine has no homeostasis mechanism, and urine can adapt to subtle and comprehensive changes, especially in the early stages of the disease. Many early disease markers in urine appear earlier than in the blood, and even earlier than the patient's symptoms, signs, and imaging pathology examinations. With the latest advances in the discovery of nano-scale extracellular vesicles called vesicles, urine vesicles have become a new source for screening and characterizing potential biomarkers. At present, a new on-disk laboratory platform has been developed. It is used to quickly and effectively isolate exosomes from urine, and potential biomarkers have been discovered, which can be used for early screening of bladder cancer. In the near future, further research on urine biomarkers will greatly promote the early diagnosis, prevention, treatment and prognosis of various diseases. Compared with plasma biomarkers, urine biomarkers are used as disease biomarkers. The early stage showed many advantages and deserves more attention and further research (Li, 2014; Jian and Youhe, 2018).

\section{Abundant Amount of Biological Information}

Urine is the mid-terminal metabolite produced by the blood after glomerular filtration, renal tubules and collecting duct reabsorption and excretion. Its composition and characteristics can reflect the body's metabolism and organ functional status. With the development of biotechnology, the rich biological information contained in urine is gradually being discovered. Millions of chemical urine tests are performed every day to identify metabolic diseases in newborns, diagnose diabetes, monitor kidney function, confirm bladder infections, detect illegal drug use, and more. At present, it is well known that the urine of normal people contains water, inorganic salts, urea, uric acid, etc., and abnormal urine can detect proteins, sugar, amino acids and ketone bodies, but the substances contained in urine are much more than these (Zha and Huang, 2019).

The David Wischett research team at the University of Alberta in Canada found a total of 3,079 chemical substances in urine. In addition to water and inorganic salts, the composition of urine also includes proteins, enzymes, nucleic acids, vitamins, hormones, amino acids and their derivatives, mesothelin, $\beta$ microglobulin, antibiotics, hormones, urokinase, mycomycin, and the final product of metabolism in the body, etc. Professor David Wischert pointed out: "Urine is an extremely complex biological fluid. We did not expect so many different chemicals to enter the urine." He said that the current urine test can only detect 16 or 7 chemicals. Ingredients, most medical textbooks only list 50-100 chemical ingredients in urine, and this study expanded the list by 30 times (Zha and Huang, 2019). The discovery of this result makes urine testing a more important method. It provides the possibility to be a test that precedes the blood test or pathological tissue section.

At present, urine biomarkers in lung cancer, cardiovascular disease, blood coagulation system, kidney cancer and other kidney diseases, bladder cancer, prostate cancer, Alzheimer's disease and autoimmune diseases have been found in the study of urine biomarkers (Huang et al., 2018). Some of the diagnostic markers or metabolites found in urine are as follows:

\section{Mesothelin}

Mesothelin is a cell surface glycoprotein and a biomarker of early pancreatic cancer. It is found in blood and urine. It has the characteristics of small molecular weight and good stability. It can also appear in cells of normal tissues, but due to its it has limited distribution in normal tissues and is highly expressed in some tumor tissues, and is expected to be used for specific treatment of tumors. Studies have shown that mesothelin is easily filtered through the glomerulus and is not easily affected by serum proteins and degrading enzymes. It is highly expressed in ovarian cancer, lung cancer, AIDS and other diseases (Wang et al., 2019).

\section{2-Microglobulin}

$\beta 2$-microglobulin is produced by nucleated cells such as lymphocytes. It is widely distributed in human urine, plasma, cerebrospinal fluid, saliva and colostrum. Under normal circumstances, the synthesis and release of $\beta 2$-microglobulin in the human body are relatively constant, which can be filtered by the glomerulus, but $99.9 \%$ will be absorbed by the proximal renal tubules, and will be degraded into amino acids in the renal tubular epithelial cells. It is used by the body and no longer flows back into the blood. Therefore, under normal circumstances, the excretion of $\beta 2$-microglobulin in urine is very small. The proximal tubule is the only place where $\beta 2$ microglobulin is processed in the body, and it is negatively correlated with tubule reabsorption rate. Therefore, it is a specific and sensitive index to evaluate the proximal tubule, and it can reflect the degree of damage. In clinical practice, 
the differential diagnosis, condition estimation, and prognostic judgment of kidney disease by measuring the content of $\beta 2$ microglobulin in blood and urine can provide valuable reference data, and $\beta 2$-microspheres can also be caused in some special clinical situations. A variety of tumors, such as chronic lymphocytic leukemia, lung cancer, kidney cancer, breast cancer, liver cancer, gastric cancer, colon cancer, rectal cancer, and lymphoma can cause $\beta 2$-microglobulin to increase in varying degrees. Fever and the use of certain drugs may also lead to different degrees of elevation, which is of reference value for the diagnosis and differential diagnosis of various diseases (Huang et al., 2018).

\section{Hormones}

At present, clinical monitoring of hormone levels in urine is often used in the detection of human chorionic pheromone in urine to detect pregnancy. Because it is convenient to test at home, it is convenient and saves the trouble of going to the hospital. Many studies now show that breast, uterus, and organs affected by hormone levels have a clear relationship between canceration and estrogen overstimulation. The metabolites of hormones are still active and have various biological effects. Some of these metabolites can inhibit the growth of cancer, and some can induce the production of cancer. When the level of hormonal metabolites exceeds that of good metabolites, it is easy to induce cancer. Therefore, monitor the level of estrogen and the level of metabolites is of great significance for the prevention of breast, ovarian, and uterine cancers. Hormones can be detected in urine using high-performance liquid chromatography-tandem technology, but for more precise detection, further improvements in testing technology are needed (Zhao, 2013).

At the same time, it has now been discovered that occupational stress hormones can be detected in urine. Occupational stress is one of the important factors leading to many diseases. It belongs to the psychological range. The evaluation of occupational stress is determined by the subjective description of patients. It is difficult to determine occupational stress. For objective quantification, some studies focused on shooters and analyzed the changes in urine indicators of shooters. It was found that vanilla mandelic acid in the urine increased significantly after stress. After studying the changes in urine indicators after exams and normal classes, it is found that vanilla mandelic acid in the urine of students after the exam has also increased (Zha and Huang, 2019). The discovery of stress markers in urine provides the possibility for future psychological diagnosis and objective quantification of occupational stress determination, which will be of great help to psychological diagnosis.

\section{Diacetylspermine}

Polyamines are low-molecular-weight aliphatic amine compounds produced in the process of metabolism. They are widely present in organisms and participate in regulating various life activities of the body, such as gene expression and translation, cell proliferation, cell apoptosis and organ development, etc. Polyamines in organisms are further divided into putrescine, cadaverine, spermidine and spermine. Diacetylspermine is a diacetylated derivative of spermine. Studies have shown that the level of diacetylspermine in the urine of a variety of cancers will be higher than normal people to different degrees. At present, it has shown a certain direction in the early diagnosis, treatment, prognosis and monitoring of recurrence of cancer $(\mathrm{Li}$ et al., 2019).

\section{Bisphenol A}

At present, people are exposed to bisphenol A (BPA) in large amounts in daily life. Animal studies have found that BPA can cause prostate cancer, but human research data is still lacking. In a study of bisphenol $\mathrm{A}$ in the urine of men, it was found that patients with prostate cancer had higher levels of BPA in their urine than those without prostate cancer. Urinary bisphenol levels are associated with prostate cancer and may have prognostic value (Prins et al., 2014; Tarapore et al., 2014).

\section{Pluripotent Stem Cells in Urine}

Using exogenous factors to transform somatic cells into induced pluripotent stem cells (iPSCs), also known as reprogramming, can be used for personalized regenerative medicine. Human induced pluripotent stem cells come from many sources, including skin (fibroblasts and keratinocytes), extra-embryonic tissues or cord blood. The reprogramming of these tissues has been achieved at different frequencies, which indicates that the cell of origin is an important determinant. The ideal cell source should be easy to obtain, susceptible and universal (any age, gender, race, and physical condition). Although human urine is a kind of biological waste, it contains a small amount of cells with self-renewal ability and differentiation potential. Urine-derived stem cells (UDSC) derived from the tortuous tubules of nephron, renal pelvis, ureter, bladder and urethra have a phenotype similar to mesenchymal stromal cells (MSC) and can be reprogrammed into induced pluripotent stem cells (iPSC). Some data indicate that urine may be the preferred source of iPSCs (Zhou et al., 2011; Bento et al., 2020).

\section{Urinary 8-Hydroxy-Deoxyguanosine}

Urine 8-hydroxy-deoxyguanosine $(8-\mathrm{OHdG})$ is a marker of DNA oxidative damage. It is mainly caused by a series of chemical processes in the body caused by ionizing radiation and chemical carcinogens, which lead to DNA oxidative damage. The markers produced are excreted in the urine through the kidneys and can only be formed through DNA oxidative damage. The content reflects the degree of oxidative damage in the body. 8 -OHdG is not only a biomarker of systemic oxidative stress, but also a risk factor for atherosclerosis, tumors, and diabetes. In clinical practice, urine 8-OHdG levels are usually used to evaluate DNA oxidative damage and oxidative stress. Studies have shown that urine The change of $8-\mathrm{OHdG}$ level may have a certain correlation with Parkinson's disease, which is not yet clear (Huang et al., 2018).

\section{Gene Fragments of Cancer}

So far, some disease markers have been found that can be found through urine tests. For example, researchers use a technology called Capp-Seq (Deep Sequencing for Cancer Individualization) to find bladder cancer DNA fragments in the urine of patients. By 


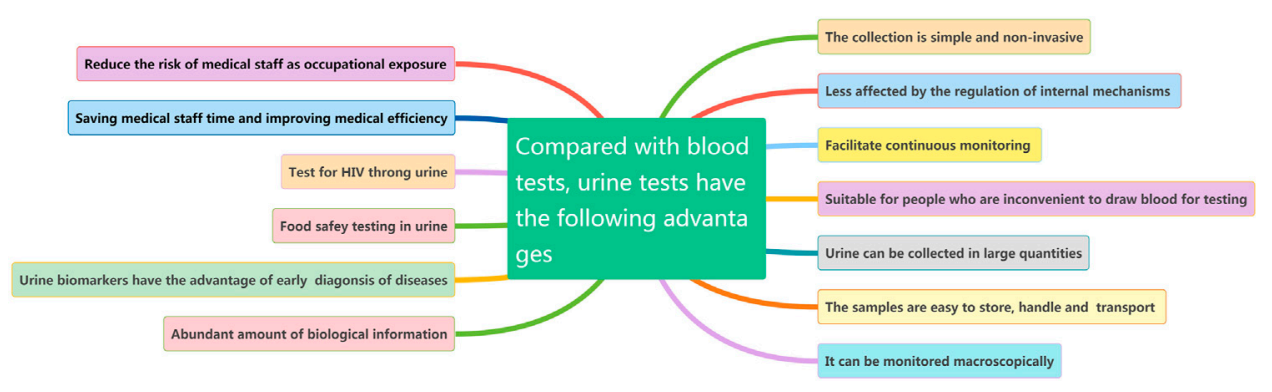

FIGURE 2 | Advantages of urine testing compared with blood testing.

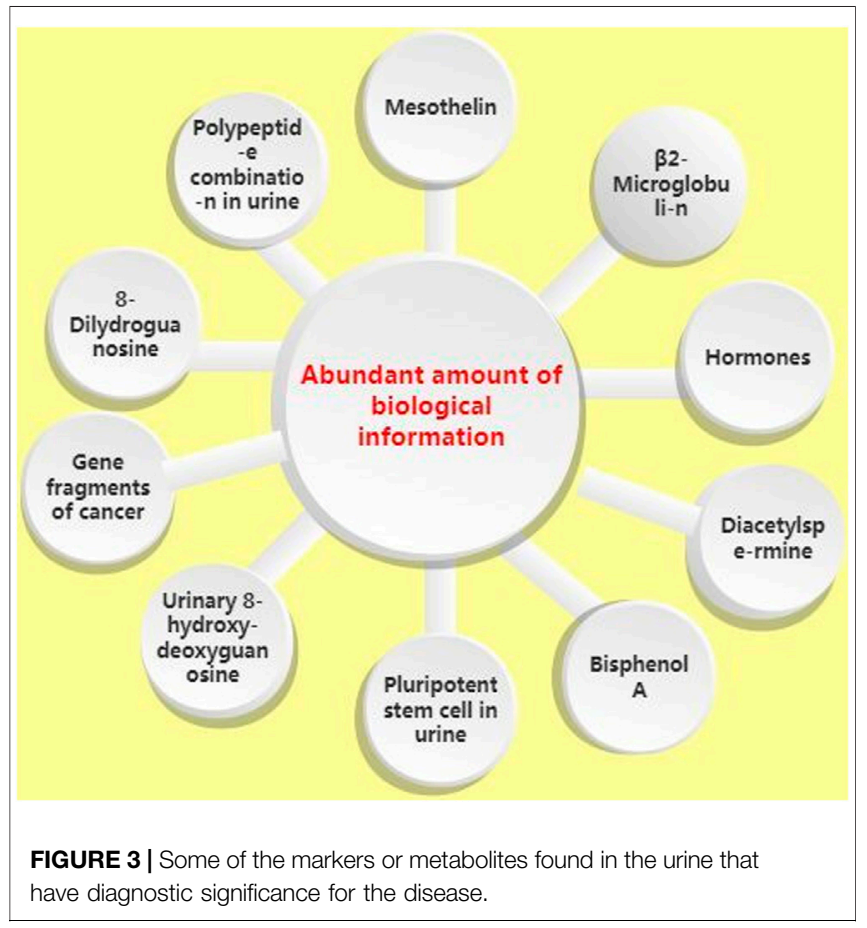

testing urine, they can effectively identify patients with early-stage bladder cancer, so that they can be treated in time. This new detection technology can accurately identify $83 \%$ of early-stage bladder cancer patients, while the current clinical urine cytology test can only identify $14 \%$ of bladder cancer patients (CJCOR, 2019). And the latest research shows that for the diagnosis and treatment of prostate cancer, a highly accurate and non-invasive biopsy method that has been developed and verified is to use 25 genomes for urine testing as a follow-up fluid for the diagnosis and treatment of prostate cancer. Which can improve the diagnosis and treatment of prostate cancer (Johnson et al., 2020).

\section{8-Dihydroguanosine (8-oxo Gsn)}

A study showed that as people grow older, 8-oxo Gsn, a substance in urine, can indicate cell damage during oxidation. A simple urine test can be used. Revealing the true age of the body, that is, showing its biological age, is expected to become a new marker of aging. The detection of such markers is more accurate than just judging by age, and may be beneficial to predict the risk of agerelated diseases (Liu and Xue, 2018).

\section{Polypeptide Combination in Urine}

Some scholars have used capillary electrophoresis-mass spectrometry (CE-MS) to compare the urine proteome of patients with coronary heart disease and normal people, and found that multiple combinations of urine peptides can identify coronary heart disease, with a sensitivity and specificity of 98 and $83 \%$. This reflects to a certain extent that the biological information carried by urine can not only directly reflect the functional state of the urinary system, but also reflect the state of blood and the entire body to a certain extent (Zha and Huang, 2019).

\section{THE UNDERSTANDING AND APPLICATION OF URINE TEST IN TRADITIONAL MEDICINE}

The microscopic analysis of urine has been one of the most routine inspection methods in modern hospitals at all levels, and it has developed to advanced microscopic technology and digital stage, but for the macroscopic characteristics of urine color, turbidity, odor, sediment and foam, etc. There are still not many ways to analyze it. Traditional urinary diagnosis is still worthy of being the most distinctive, simplest and most effective diagnostic method for Tibetan medicine, Mongolian medicine and Chinese medicine.

Due to the convenient availability of urine, Urinary diagnosishas an important position in traditional medicine. In the consultation of traditional Chinese medicine, the consultation of urine status is an indispensable link, as reflected in the "Ten Questions" of Chinese medicine. Traditional Chinese medicine believes that urine is one of the final products of body fluid metabolism. Water enters the body, transports and transforms through the spleen and stomach, and is transferred to the lungs. Through lung qi disseminate and liquefy, the esseatence part of it is used to nourish the whole body, and the turbid fluid in the metabolism of the viscera is transferred to the kidney or bladder. Under the action of the transpiration and gasification of the kidney qi, the clearer is dispersed throughout the body. Turbidity turns into urine. Urine contains the turbid fluid of various viscera 
metabolism and is regulated by multiple viscera. If the urine is abnormal, it reflects the abnormal function of the internal organs. The excrement of the human body is the messenger of physiological and pathological changes in the body. Su Weng, Zhi Zheng, Yao Da Lun records: "the body has an antagonist reflex, and the water is turbid, which is related to heat in the body. The body fluids in the course of the disease are cold and clear, which is a manifestation of body cold," in other words, the cold and hot nature of the disease can be determined by the effluent (Yang et al., 2016). For some exogenous diseases, it is sometimes difficult to distinguish whether the pathogen is on the outside or inside at this time. Zhongjing Zhang pointed out in Shang Hang Lun that it can be distinguished by urine conditions, such as Article 56: "Patients with Febrile Diseases and no bowel movements for six or 7 days, headache and fever syndrome, should be given Chengqi Decoction. If the patient has cold urine and knows that the patient's pathogen is not in the body but still outside the body, sweating should be given. If the headache is accompanied by nose bleeding, Suitable for taking Guizhi Tang." By judging whether the urine is hot or cold. Identify whether the disease is on the outside or on the inside (Li and Guo, 1997), and the profit and loss of body fluid in the body can be judged by changes in urine output and urinary patency. During the development of the disease, the change from unfavorable urination to good urination also marks the transformation of the disease to the better, and the prognosis is good (Wang et al., 2009). Combining the information of the other four medical examinations to make the dialectic of the outside and inside, cold and heat, deficiency and excess, yin and yang, through the observation and analysis of the patient's internal and external conditions, to determine the location of the patient's disease, its stage and prognosis, etc., (Zhang et al., 2020). Tibetan doctors also have unique insights on urinary diagnosis and have summarized a set of relatively complete urine collection, observation and diagnosis methods. Among the various traditional medical systems in the world, there is no other medical system whose urinary consultation content and the degree of detailed observation can compare with the urinary consultation of Tibetan medicine (Yang et al., 2016). The Tibetan medicine urinary clinic has also been included in the first batch of intangible cultural heritage lists of the country. In the History of World Civilization, it was recorded: "Urinalysis was a popular method at the time. The doctors in Tibet believe that there is no need to observe any other parts of the patient, just check the patients urine to treat the patient." It can be seen that urinary diagnosis plays a pivotal role in Tibetan medicine (Tang et al., 2020). Tibetology believes that urine belongs to the category of metabolites. It is the dregs of water throughout the body. It is formed by continuous differentiation after the human body consumes food (Wang et al., 2009; Renqing, 2016). The source of urine is mainly produced by the five vital and six vessel organs. Urine the changes are related to living environment, diet and disease. The contents of Tibetan medicine urinary consultation mainly include smelling smell, observing color, how quickly the steam disappears, the size of steam, floating matter on the surface of urine, urine sediment, flocculent and foam in the middle layer of urine. For frail patients, Tibetans sometimes put the patient's urine in a special container for Tibetan doctors to diagnose, so as to save the patient from the hardships of traveling on the road. The urinary clinic has a good reputation and practice foundation among the Tibetan people (Bu and Ram, 2011; Shaoju, 2019).

\section{THE APPLICATION OF URINE TESTING TECHNOLOGY IN SOCIAL PRODUCTION AND LIFE}

Urine testing technology is also widely used in social life. In daily life, urine can be tested by pregnancy test sticks to determine whether pregnancy or not. The principle is to detect whether the urine contains human chorionic gonadotropin (HCG). The urine can be used to monitor whether you are pregnant or not, and the results can be obtained within a few minutes, eliminating the need to go to the hospital to queue for blood tests, and you can easily operate at home, which is safe and non-invasive. The test can go to the hospital for related examinations after a positive result is made for further confirmation, saving manpower, material resources and financial resources, and improving efficiency.

Since 1968, the International Olympic Committee has been using urine tests to detect whether athletes are taking stimulants. Urine tests are the main method and blood tests are supplemented. By collecting blood for testing, there may be discomfort after blood sampling or secondary adverse reactions, which may affect the results of the game. Urine tests are used to identify whether or not to take doping, which reduces the risk of exposure of athletes, improves the efficiency of testing, and minimizes the probability of these adverse events caused by invasive testing. It is a simple, effective and highly accurate method to detect whether athletes are taking doping through urine testing (CSC, 1995; Zhou et al., 2015). In the past, it was impossible to find out after 2 weeks of stopping doping. With the advent of high-resolution mass spectrometers, the detection technology has made a great leap and development. Now even if the interval is 50-60 days, it can be detected relatively easily.

My country as a major drug ban country, it is also one of the methods of anti-drug inspection to test whether the urine contains drug metabolites to detect whether drugs have been used. Compared with blood tests, urine tests are more convenient for large-scale screening. Among drug users, the probability of carrying AIDS is relatively high, and the use of urine tests also reduces the risk of exposure of medical staff and related staff to a certain extent.

There is also the use of HP-ELISA diagnostic reagent urine test for the diagnosis of HP (Helicobacter pylori) infection (Yixin and Jinbo, 2014). Urine tests are used to assess whether there has been recent alcohol consumption. By using $\mathrm{uEtG}$ as a biomarker of alcohol, it can help predict alcohol intake and overcome the deficiencies of self-reported. It can be used to help people who quit alcohol or researchers. Observation research during the experiment (Grodin et al., 2020). There is also the use of commercial kits to extract and determine human DNA from urine. Urine may be successfully genotyped even if it is stored at $20^{\circ} \mathrm{C}$ for several months, and urine can be collected at many times of the day. To, this has important implications for public health 
research (Bali et al., 2014). In the current use situation, there is also the use of test paper to detect albumin in the urine to monitor the occurrence and progress of cardiovascular disease and kidney disease (Smink et al., 2012; Aiumtrakul et al., 2021). It is believed that there will be more urine testing technologies in the future to improve the convenience of people's production and life and promote social development.

\section{THE ADVANTAGES AND DISADVANTAGES OF THE COMMON URINE TESTING TECHNOLOGIES}

At present, the clinically common urine detection techniques include microscopy, urine analyzer detection and urine dry chemical detection. The advantage of microscopy is that the detection accuracy is relatively high, but the detection time is long and the work efficiency is lower, and the requirements for the inspection personnel's operating technology and inspection skills are also relatively high (Shaoju, 2019). The advantages of urine dry chemical analysis and detection are simple and fast operation, and only a small amount of urine is needed to obtain a number of experimental parameters. However, it has strong randomness, many interference factors, and more frequent shortcomings of false positives and false negatives (Jingzhi, 2007). The principle of analysis and detection of urine sediment analyzer is basically similar to the principle of manual microscopy. Both are intuitive observations of the constituents in the urine. However, the urine sediment analyzer undergoes strict timing, fixed speed, and quantification to quantify the test results, but the disadvantage is that the automatic urine sediment analyzer is susceptible to the influence of bacteria, myoglobin, and heat-prone enzyme factors, which reduces the specificity (Yuhang et al., 2021).

\section{DIFFICULTIES FACED BY THE DEVELOPMENT OF URINE TESTING TECHNOLOGY}

With the development of science and technology and the advancement of human understanding, the technology of urine testing has made great breakthroughs, but there is still a large distance from the ideal test target. In the urine collection process, it is also affected by many factors such as the patient's collection time and improper operation during the collection process, resulting in test errors (Shi, 2020; Zhou, 2020). The complexity and diversity of urine components and the susceptibility of urine to various factors make the research of urine still a great challenge at present. The more factors that affect urine, the more samples are needed for research and analysis, the amount is larger. At present, people are more enthusiastic about blood research than urine. From the perspective of the number of papers published, urine biomarkers only account for $7 \%$ of blood (Zha and Huang, 2019), which also reflects the impact on urine.
The research investment is far less than the research on blood. These are the problems currently facing the further development of urine testing technology. Therefore, opportunities and challenges coexist.

\section{PROSPECTS AND FOREGROUND FOR THE FUTURE DEVELOPMENT OF URINE TESTING}

Urine is rich in biological information. In the era of rapid development of bioanalysis technology and big data processing information, the human health code contained in the complex urine components will also be continuously decrypted. Some researchers believe that the method of searching for cancer DNA in body fluids rather than blood may be more widely used. Now for colon cancer, prostate cancer and other new generation urine diagnostic technologies, and some signs of finding signs in urine the better than the markers in the blood has also been initially recognized and explored by people. The technology of detecting cancer information markers through urine has entered the development process or will soon enter the hospital ( $\mathrm{Li}$ et al., 2020). Some scholars also want to use urine to detect volatile organic compounds to achieve the purpose of distinguishing cancer types (Bannaga et al., 2020). Some experiments have proved that the health of athletes can be monitored through urine observation and analysis, so as to protect the health of athletes and improve their physical fitness (Pero et al., 2020), which also indicates whether ordinary healthy people can also perform health self-examination through urine. Accessibility makes it easier for people to monitor their physical health (Liu et al., 2020; Liu et al., 2021). Today, with the continuous development of medical inspection technology, these are all possible ideas. If more researchers can participate in the mining of the potential gold mine of urine testing, it may greatly accelerate the development of medical laboratory science and change the face of medical research and medical practice in the next century.

\section{AUTHOR CONTRIBUTIONS}

$\mathrm{HZ}$ and $\mathrm{XR}$ contributed to conception and supervised the project. $\mathrm{ZZ}, \mathrm{JL}, \mathrm{YC}$, and JC contributed in doing literature searches and wrote the manuscript draft. HZ, XR, ZZ, JL, YC, and JC equally revised and approved the manuscript. All authors have read and approved the final draft manuscript.

\section{FUNDING}

This work was supported by National Natural Science Foundation of China (81973697), project leader: HZ and National Natural Science Foundation of China (81774448), project leader: XR. 


\section{REFERENCES}

Aiumtrakul, N., Phichedwanichskul, K., Saravutthikul, S., Ottasat, K., Visuthitepkul, K., Jaruthiti, T., et al. (2021). Urine Albumin Dipstick Independently Predicts Cardiovascular and Renal Outcomes Among Rural Thai Population: a 14-year Retrospective Cohort Study. BMC Nephrol. 22 (1), 18. doi:10.1186/s12882-020-02215-8

Bali, L. E., Diman, A., Bernard, A., Roosens, N. C. H., and Keersmaecker, S. J. D. E. (2014). Comparative Study of Seven Commercial Kits for Human DNA Extraction from Urine Samples Suitable for DNA Biomarker-Based Public Health Studies. J. Biomol. Tech. : JBT 25 (4), 96-110. doi:10.7171/jbt.142504-002

Bannaga, A. S. I., Kvasnik, F., Persaud, K. C., and Arasaradnam, R. (2020). Differentiating Cancer Types Using a Urine Test for Volatile Organic Compounds. J. Breath Res. 15, 2-10. doi:10.1088/1752-7163/abc36b

Bento, G., Shafigullina, A. K., Rizvanov, A. A., Sardão, V. A., Macedo, M. P., and Oliveira, P. J. (2020). Urine-Derived Stem Cells: Applications in Regenerative and Predictive Medicine. Cells 9 (3), 573. doi:10.3390/cells9030573

Bouatra, S., Aziat, F., Mandal, R., Guo, A. C., Wilson, M. R., Knox, C., et al. (2013). The Human Urine Metabolome. PLOS ONE 8 (9), e73076. doi:10.1371/ journal.pone.0073076

$\mathrm{Bu}, \mathrm{L}$., and Ram, L. (2011). On the Urinary Diagnosis of Tibetan Medicine[J]. Med. Inf. 24 (03), 862-863. doi:10.3969/j.issn.1006-1959.2011.03.065

Carrera, O. H., and Bueno, M. D. M. J. (2020). Cost Analysis of the Automated Examination of Urine with the Sysmex UN-series in a Spanish Population. PharmacoEconomics Open 4, 605-613. doi:10.1007/s41669-020-00200-3

CJCOR The Birth of a New Urine Detection Technology for Bladder Cancer[J]. Chin. J. Clin. Oncol. Rehabil. 2019, 26 (12), 1516.

CSC (1995). Urine Test and Blood Test [J]. China Sports Coach 01, 36.

Dai, J., Li, Q., Qin, S., Qin, S., Zhang, L., Min, M., et al. (2003). Comparative Analysis of HIV Antibody Test Results in Blood and Urine of 203 Drug Addicts [J]. China AIDS and STDs (05), 278-279. doi:10.3969/j.issn.1672-5662.2003.05.009

Fan, X. (2020). Influencing Factors and Control Measures of Blood Test Results [J]. Guide China Med. 18 (31), 120.

Gao, Y. (2013). Urine May Be a Better Source of Biomarkers than blood[R]. Shandong, China: Qingdao.

Grodin, E. N., Nguyen, X. T., Ho, D., Bujarski, S., and Ray, L. A. (2020). Sensitivity and Specificity of a Commercial Urinary Ethyl Glucuronide (ETG) Test in Heavy Drinkers. Addict. Behav. Rep. 11, 100249. (prepublish). doi:10.1016/ j.abrep.2020.100249

Hexing, W., Bin, W., Qi, Z., Zhao, Y., Fu, C., Femg, X., et al. (2015). Antibiotic Body burden of Chinese School Children: a Multisite Biomonitoring-Based Study. Environ. Sci. Technol. 49 (8), 5070-5079. doi:10.1021/es5059428

Huang, S., Xiaobin, T., Hui, C., and Zha, Y. (2018). Urine Marker Science[M], 6. Beijing, China: Science Press, 77.

Jian, J., and Youhe, G. (2018). Urine Biomarkers in the Early Stages of Diseases: Current Status and Perspective. [J]. Discov. Med. 25 (136).

Jingzhi, M. (2007). Clinical Evaluation of the Comprehensive Application of Multiple Methods in 600 Urine Tests[J]. Cliniques (19), 1435.

Johnson, H., Guo, J., Zhang, X., Zhang, H., Simoulis, A., Wu, A. H. B., et al. (2020). Development and Validation of a 25-Gene Panel Urine Test for Prostate Cancer Diagnosis and Potential Treatment Follow-Up. BMC Med. 18, 376. doi:10.1186/ s12916-020-01834-0

Li, S., and Guo, S. (1997). Significance of Clinical Application of "Treatise on Febrile Diseases" [J]. Guangming Traditional Chin. Med. 12 (73), 5-6.

Li, N., Ho, K. W. K., Ying, G.-G., and Deng, W.-J. (2017). Veterinary Antibiotics in Food, Drinking Water, and the Urine of Preschool Children in Hong Kong. Environ. Int. 108, 246-252. doi:10.1016/j.envint.2017.08.014

Li, J., Yang, M., Cheng, Y., Sun, T., and Liu, J. (2019). The Clinical Value of $\mathrm{N} \sim 1, \mathrm{~N} \sim(12)$-diacetylspermine as a New Tumor Marker[J]. Tianjin Sci. Technol. 46 (12), 85-88. doi:10.14099/j.cnki.tikj.2019.12.026

Li, F., Chen, M., Zhang, Y., Yan, B., and Zheng, J. (2020). Multi-factor Analysis of Urine NMP22 Positive and Urine Routine Examination and Blood Examination in Zhoupu Area of Shanghai. Lab. Med. Clin. 17 (11), 1515.

Li, M. (2014). Urine Is an Ideal Source of Disease Markers [D]. Beijing, China: Peking Union Medical College.
Liu, X., and Xue, H. (2018). Disease-related Information Is Hidden in Urine [J]. Basic Med. Clin. 38 (12), 1748. doi:10.16352/j.issn.1001-6325.2018.12.020

Liu, J. N., Tidwell, T., Zhao, H. H., Ren, Q. J., Mao, M., Li, J. L., et al. (2020). Theoretical Characteristics of Tibetan Medicine. World J. Tradit Chin. Med. 6, 490-499. doi:10.4103/wjtcm.wjtcm_21_20

Liu, J., Zhang, Z., Pang, X., Cheng, Y., Man, D., He, X., et al. (2021). Analysis of the Distribution of Urine Color and Its Relationship With Urine Dry Chemical Parameters Among College Students in Beijing, China - A Cross-Sectional Study. Front. Nutr. 8, 719260. doi:10.3389/fnut.2021.719260

Pero, R., Brancaccio, M., Mennitti, C., Gentile, L., Arpino, S., De Falco, R., et al. (2020). Urinary Biomarkers: Diagnostic Tools for Monitoring Athletes' Health Status. Int. J. Environ. Res. Public Health 17, 2-14. doi:10.3390/ijerph17176065

Prins, G. S., Hu, W.-Y., Shi, G.-B., Hu, D.-P., Majumdar, S., Li, G., et al. (2014). Bisphenol A Promotes Human Prostate Stem-Progenitor Cell Self-Renewal and Increases In Vivo Carcinogenesis in Human Prostate Epithelium. Endocrinology 155 (3), 805-817. doi:10.1210/en.2013-1955

Qiu, W., Long, W., Luo, H. (2020). Clinical Application of Urine Test in the Diagnosis of Acute Fever Caused by Legionella pneumophila in Children. Heilongjiang Med.. 9 (44), 1252. doi:10.3969/j.issn.1004-5775.2020.09.033

Ren, Y. (2020). Feasibility Study of HIV Antibody Rapid Detection Method for SelfDetection [D]. Beijing, China: Chinese Center for Disease Control and Prevention.

Renqing, W. M. (2016). Discussion on the Application of Metabolomics Technology in the Urinary Diagnosis of Tibetan Medicine [J]. Chin. J. Ethnic Med. 22 (09), 39-50. doi:10.16041/j.cnki.cn15-1175.2016.09.027

Shaoju, S. (2019). Analysis of the Application Value of Urine Analyzer and Microscopy in Urine Testing[J]. China Med. Device Inf. 25 (09), 160-161. doi:10.3969/j.issn.1006-6586.2019.09.075

Shi, G. (2020). How Much Do You Know about Urine Tests [N]. Nanning City: Guangxi Zhuang Autonomous Region, 033.

Smink, P. A., Lambers Heerspink, H. J., Gansevoort, R. T., de Jong, P. E., Hillege, H. L., Bakker, S. J., et al. (2012). Albuminuria, Estimated GFR, Traditional Risk Factors, and Incident Cardiovascular Disease: the PREVEND (Prevention of Renal and Vascular Endstage Disease) Study. Am. J. Kidney Dis. 60 (5), 804-811. doi:10.1053/j.ajkd.2012.06.017

Tang, Z., Wu, A., Huang, X., Xiong, X., Li, C., Gong, R., et al. (2020). Investigation on Infectious Occupational Exposure of Medical Staff in Xiangya Hospital [J]. Chin. J. Nosocomial Infect. 30 (18), 2864. doi:10.11816/cn.ni.2020-192881

Tarapore, P., Ying, J., Ouyang, B., Burke, B., Bracken, B., and Ho, S. M. (2014). Exposure to Bisphenol A Correlates with Early-Onset Prostate Cancer and Promotes Centrosome Amplification and anchorage-independent Growth In Vitro. PLOS ONE 9 (3), e90332. doi:10.1371/journal.pone.0090332

Wang, H., and Bao, Z. (2019). Preliminary Study and Application of Hormone Analysis Methods in Urine of Diapers[J]. Environ. Occup. Med. 36 (11), 1001-1006. doi:10.13213/j.cnki.jeom.2019.19324

Wang, J., Gu, S., Zang, Y., Chen, B., and Shao, L. (2009). Looking at the Potential Value of Metabolomics in the Standardization of TCM Syndromes from Traditional Medical Urinary Diagnosis [J]. World Sci. Technol. 11 (01), 24-35. doi:10.3969/j.issn.1674-3849.2009.01.010

Wang, H., Tang, C., Yang, J., Wang, N., Jiang, F., Xia, Q., et al. (2018). Predictors of Urinary Antibiotics in Children of Shanghai and Health Risk Assessment. Environ. Int. 121 (Pt 1), 507-514. doi:10.1016/j.envint.2018.09.032

Wang, Y., Wang, J., Zhang, Z., and Chen, B. (2019). Urine Mesothelin Combined with Human Epididymal Protein in Patients with Ovarian Tumors and its Clinical Significance[J]. Shaanxi Med. J. 48 (8), 1088-1090.

Yang, M., Duan, X., and Shou, H. (2016). The Role of Urine Appearance in the Diagnosis of Traditional Chinese Medicine [J]. Guide Chin. Med. 22 (15), 114-116. doi:10.13862/j.cnki.cn43-1446/r.2016.15.040

Ye, L., Chen, X., Su, L., Liang, S., Yang, H., Yuan, D., et al. (2019). Comparison of the Performance of HIV Antibody Non-invasive (Oral Mucosal Exudate and Urine) Testing Reagents and Invasive (Blood) Testing Reagents[ J]. Int. J. Lab. Med. 40 (10), 1169-1173. doi:10.3969/j.issn.1673-4130.2019.10.005

Yixin, S., and Jinbo, Q. (2014). Evaluation of Non-invasive Laboratory Diagnosis Methods for Helicobacter pylori Infection[J]. Chin. J. Endemic Dis. Control. 29 (S2), 116.

Yuan, L., Li, Y. R., and Xu, X. D. (2018). Chronobiology -2017 Nobel Prize in Physiology or Medicine. Yi Chuan 40 (01), 1-11. doi:10.16288/j.yczz.17-397 
Yuhang, C., Mingqin, F., and Hong, H. (2021). Application of Automatic Urine Sediment Analyzer and Microscope Detection Method in Routine Urine Inspection[J]. Med. Equipment 34 (17), 59-63.

Zha, Y., and Huang, S. (2019). Urine Biochemistry and Inspection[M]. Beijing, China: People's Medical Publishing House, 5-815.

Zhang, D., and Liang, Y. (2016). Blood[M]. Wuhan, China: Hubei Science and Technology Press, 11-12.

Zhang, Y., Bai, J., and Zhou, Y. (2020). Characteristics of Occupational Exposure to Blood-Borne Pathogens in a Hospital in Southwest China from 2015 to 2019 [J]. Chin. J. Infect. Control. 19 (12), 1054. doi:10.12138/j.issn.16719638.20206169

Zhao, H. (2013) Study on the Correlation Between Estrogen Metabolites in Liquid and Endometrial Cancer [J]. Hebei Medical University.

Zhou, T., Benda, C., Duzinger, S., Huang, Y., Li, X., Li, Y., et al. (2011). Generation of Induced Pluripotent Stem Cells from Urine. Jasn 22 (7), 1221-1228. doi:10.1681/asn.2011010106

Zhou, T., Wang, M., Fang, H., Cui, J., Zhang, J., Zhuo, Z., et al. (2015). Research and Implementation of Doping Detection Methods[J]. Rev. Develop. Sci. Technol. Award China Assoc. Anal. Test. 9.
Zhou, T. (2020). What Is in Your Urine? [J]. Healthy Home 11, 57.

Conflict of Interest: The authors declare that the research was conducted in the absence of any commercial or financial relationships that could be construed as a potential conflict of interest.

Publisher's Note: All claims expressed in this article are solely those of the authors and do not necessarily represent those of their affiliated organizations, or those of the publisher, the editors and the reviewers. Any product that may be evaluated in this article, or claim that may be made by its manufacturer, is not guaranteed or endorsed by the publisher.

Copyright (C) 2022 Zhang, Liu, Cheng, Chen, Zhao and Ren. This is an open-access article distributed under the terms of the Creative Commons Attribution License (CC $B Y)$. The use, distribution or reproduction in other forums is permitted, provided the original author(s) and the copyright owner(s) are credited and that the original publication in this journal is cited, in accordance with accepted academic practice. No use, distribution or reproduction is permitted which does not comply with these terms. 